FORMATION Formation emploi

Revue française de sciences sociales

119 | juillet-septembre 2012

pêle-mêle

\title{
Entre relation d'emploi et relation de services : Les stratégies de deux sociétés de services en ingénierie informatique (SSII)
}

Between employment relations and service relations: the strategies of two

information technology service firms

Zwischen Beschäftigungsverhältnis und Dienstleistungsverhältnis: die

Strategien zweier Systemhäuser

Entre relación de empleo y relación de servicios : Las estrategias de dos empresas de servicios en ingeniería informática (SSII)

\section{Anne Dietrich et Françoise Lozier}

\section{(2) OpenEdition}

\section{Journals}

Édition électronique

URL : http://journals.openedition.org/formationemploi/3692

DOI : 10.4000/formationemploi.3692

ISSN : 2107-0946

\section{Éditeur}

La Documentation française

Édition imprimée

Date de publication : 10 septembre 2012

Pagination : 29-46

ISSN : 0759-6340

Référence électronique

Anne Dietrich et Françoise Lozier, «Entre relation d'emploi et relation de services : Les stratégies de deux sociétés de services en ingénierie informatique (SSII) », Formation emploi [En ligne], 119 | juilletseptembre 2012, mis en ligne le 26 octobre 2012, consulté le 30 octobre 2020. URL : http:// journals.openedition.org/formationemploi/3692 ; DOI : https://doi.org/10.4000/formationemploi.3692 


\title{
Entre relation d'emploi et relation de services:
}

\section{Les stratégies de deux sociétés de services en ingénierie informatique (SSII)}

\begin{abstract}
AnNe Dietrich
Maître de conférences, IAE (Institut d'administration des entreprises) de Lille, UMR-

CNRS 8179
\end{abstract}

FRANÇOISE LOZIER

Maître de conférences, université Dauphine, IRISSO (Institut de recherche interdisciplinaire en

sciences sociales) UMR 7170

Résumé

Entre relation d'emploi et relation de services : Les stratégies de deux sociétés de services en ingénierie informatique (SSII)

Les SSII (sociétés de services en ingénierie informatique) constituent un champ d'observation pertinent pour interroger les effets de la relation de service sur la relation d'emploi. Nous nous appuyons sur l'étude comparée de deux SSII qui, malgré des choix de développement différents, parviennent à fidéliser leurs salariés. Il s’agit de montrer que les pratiques d'emploi largement contestées des SSII sont en fait liées aux modes de prestations que leur imposent les clients et aux stratégies que, sur ces bases, elles développent.

Mots clés : Relation de service, SSII - société de service informatique, pratique de GRH

Abstract

Between employment relations and service relations : the strategies of two information technology service firms

Information technology services firms are a field of observation relevant to examine the effects of service relations on employment relations. Based on a comparative study of two firms which succeed in retaining their employees, despite different strategic development choices, we show that employment practices, widely disputed in these firms, are in fact 
related to the services demanded by their customers and to the strategies that they develop on this basis.

Key words: Service relationship, software engineering company, HR management practice

Journal of Economic Literature: M 51, M 54, M 55

Traduction : Auteurs

Les sociétés de services en ingénierie informatique (SSII) constituent un champ d'observation pertinent pour interroger les transformations de la relation d'emploi, et plus précisément les effets de la relation de service sur la relation d'emploi. Les raisons en sont multiples. Sur un marché en croissance continue, porté par l'innovation et le renouvellement permanent des technologies, les SSII sont fortement pourvoyeuses d'emplois et réalisent $90 \%$ des recrutements de cadres informaticiens (Apec, 2006). Elles constituent un archétype de la relation de service et de la marchandisation d'une production immatérielle : prestataires de services hautement qualifiés, elles mettent leurs salariés à la disposition des entreprises clientes utilisatrices d'informatique et tirent de leur travail l'essentiel de leur valeur ajoutée. Leurs modes de fonctionnement font de ces travailleurs des hautes technologies et du conseil la figure emblématique d'un salarié indépendant, employable et mobile (Boltanski, Chiapello, 1999 ; Gazier, 2006 ; Zune, 2006). Leurs pratiques d'emploi sont toutefois controversées. Elles génèrent un turnover qui, au gré des marchés, tantôt leur convient, tantôt devient contre-productif et les contraint à s'interroger sur leurs stratégies en matière de gestion des ressources humaines mais aussi de service au client.

Lobjectif de cet article est de comprendre la spécificité des conditions d'emploi et de travail des informaticiens et d'analyser le lien entre pratiques d'emploi et stratégies des SSII. Pour cela, nous abordons le fonctionnement des SSII à partir de leurs pratiques de gestion. Ce secteur est dominé par le travail en régie. Il désigne à la fois un mode de prestation de service et une pratique d'emploi consistant à fournir à l'entreprise cliente les collaborateurs correspondant aux profils qu'elle a définis et qui travailleront dans ses locaux pour une durée déterminée. Dans ce cadre, la SSII n’a qu'une obligation de moyens et son activité s'apparente à un prêt de main-d'œuvre dont la licéité est régulièrement contestée.

À côté de cette modalité existe un autre type de prestation, le forfait. Il consiste à vendre et réaliser une "solution client $a d$ hoc ", sur la base d'une somme forfaitaire préalablement négociée avec le client. Le travail au forfait s’apparente davantage à une activité de sous-traitance. La SSII participe à la définition du besoin du client, le conseille mais reste maître d'œuvre du projet et s'engage sur les moyens, délais et résultats.

La première partie de l'article présente les spécificités des SSII, leur dépendance à l'égard des entreprises clientes, les modes de fonctionnement qui en découlent et les pratiques 
d'emploi qui en font des lieux de passage. Une seconde partie propose un contre-exemple. Elle rend compte de l'étude empirique de deux SSII qui, malgré des stratégies de développement différentes, ont fait le choix d'investir dans les ressources humaines.

\section{Encadré 1 \\ Méthodologie}

L'étude s'appuie sur :

- des recherches documentaires récentes sur les SSII (sociétés de services en ingénierie informatique), la consultation de sites et revues de la profession, la littérature en sciences sociales sur les transformations du marché et des métiers informatiques, l'évolution des formes d'emploi et d'organisation ;

- des entretiens exploratoires avec des DRH (directeurs des ressources humaines) de grandes SSII ou de leurs filiales (5) et les dirigeants de deux petites SSII ;

- la réalisation de deux monographies à partir du suivi longitudinal sur une durée de 3 ans de deux SSII (I et N), d'entretiens semi-directifs avec différents acteurs : dirigeant de N ( $2 \mathrm{~h}$ : stratégie, vision du marché, métiers) ; deux responsables de recrutement de $\mathrm{N}$ (1 h : (vision du marché du travail, comportements des candidats, modalités de recrutement) ; DRH I et N (quatre à cinq entretiens de 2 à $3 \mathrm{~h}$, pour chaque entreprise : pratiques de $\mathrm{GRH}$, emplois) ; responsable de recrutement de I (deux entretiens de 1h 30), cinq informaticiens de I ( $2 \mathrm{~h}$ : conditions de travail, métiers) ;

- la consultation de documents internes (rapports d'activité, enquêtes de satisfaction, benchmarks internes, presse, trophées).

Les matériaux récoltés ont donné lieu à une analyse comparée des deux SSII qui soulignent, au-delà de leurs différences (taille, métier), des choix stratégiques et des pratiques de GRH analogues.

\section{Les SSII : lieux de passage}

Acteur essentiel du marché de l'emploi informatique, les SSII ne sont souvent qu'un lieu de passage pour les informaticiens. Une revue des travaux consacrés aux SSII permet d'identifier des contextes et des pratiques d'emploi qui fragilisent le lien salarial ; et ce en raison, notamment, de la triangulation des relations de travail qui résulte de la mise à disposition de salariés chez le client.

\subsection{Des travaux focalisés sur le système d'emploi informatique}

Les travaux consacrés aux SSII se sont focalisés sur le système d'emploi informatique, en économie, sur le groupe professionnel et la professionnalité des informaticiens, en sociologie (Lanciano-Morandat, 1996). Tous soulignent la spécificité du profil d'ingénieur en SSII : jeune, masculin (Fondeur, Sauviat, 2003), "orienté marché » (Gazier, 2006), 
"nomade» (Zune, 2006), "Plaque tournante des flux de recrutements ». Sur le marché informatique des années 2000 (Poussou-Plesse et al., 2009), les SSII sont «au cour du système d'emploi informatique" (Fondeur, Sauviat, 2003, p. 107) ; en outre, le marché des services connaissant une croissance exponentielle depuis $1976^{1}$, elles sont pourvoyeuses d'emploi : 150000 emplois très qualifiés créés ces dix dernières années ${ }^{2}$. Elles offrent aux jeunes diplômés l'opportunité d'acquérir une expérience à travers diverses missions et d'accéder aux grandes entreprises utilisatrices d'informatique qui demeurent le principal employeur d'informaticiens (Fondeur, Sauviat, 2003).

Cette place particulière des SSII s'explique par leurs conditions d'émergence, en position d'intermédiaire entre les constructeurs de matériels informatiques qui dominent le secteur dans les années 60 et les entreprises utilisatrices, confrontées à de multiples problèmes auxquels les constructeurs n'apportent pas de réponse. Les SSII prennent le relais et comblent "le vide laissé par les constructeurs " (Poussou-Plesse et al., 2010, p. 23) en offrant une palette de services aux entreprises. Issues du recentrage sur le client (Liaroutzos, Robichon, 1999) ${ }^{3}$, elles se spécialisent et se professionnalisent. Leur champ d'activités s'étend, le terme d'ingénierie se substitue à celui de conseil pour intégrer une pluralité de services allant du conseil et de l'ingénierie (conception et mise en œuvre du système informatique) à l'infogérance (prise en charge à distance de la fonction informatique), en passant par la maintenance et l'édition de logiciels. Les SSII constituent une " pièce maîtresse " du marché informatique ; en effet, elles prennent en charge tout ou partie de la fonction informatique d'une entreprise, externalisée en raison du niveau d'expertise requis, du renouvellement rapide des connaissances, des difficultés de recrutement afférentes, de la possibilité sur un plan comptable d'imputer les dépenses réalisées en investissements de " recherche et développement " plutôt qu'en charges salariales.

Le nombre de SSII explose entre 1980 et 1998, expliquant la dispersion du marché dominé par les $\mathrm{TPE}^{4}$; en effet, créer une SSII ne requiert aucun investissement capitalistique et peut s'avérer rentable. Mais les vingt deux plus grandes SSII, en France, représentent près de $50 \%$ du marché total des services et les quatre premières $20 \%$ de ce marché 5 . Celui-ci arrive à maturité et tend à se concentrer sous la pression des clients qui "réduisent le nombre de leurs partenaires ${ }^{6}$ » et développent, depuis 2002, des pratiques de référencement dras-

1. «Le marché des services informatiques sort du lot », Les Echos, 03/08/2010, http://entrepreneur.lesechos. fr.

2. Source : bilan Syntec Informatique, 2008.

3. À partir des années 80 , une évolution majeure accompagne les progrès technologiques décisifs en matière d'informatique, à savoir l'apparition d'organisations centrées sur le client et l'utilisateur.

4. 21300 entreprises emploient plus de 370000 salariés, dont 4700 de plus de 10 salariés : Bilan Syntec Informatique 2008.

5. Source IDC France, Les parts de marché des sociétés de services, mai 2009.

6. "Renault est ainsi passé de 300 fournisseurs de services informatiques à trois, France Télécom de 1000 à 300 ", (Syntec, 2005). 
tiques (réduction des coûts, des durées de missions). La concurrence est telle que fusions et acquisitions se multiplient. Dès lors, il existe une forte interdépendance entre entreprises clientes et SSII dont l'activité dépend de la conjoncture et de la situation économique des clients qui, eux-mêmes, font varier leurs effectifs informatiques en fonction des services offerts par les SSII. Berrebi-Hoffmann (2006) définit le business model dominant des SSII comme la mise en adéquation de flux de main-d'œuvre et de flux de commandes. Le recrutement constitue donc un enjeu majeur pour les SSII (Baret, Everaere, 2002) ; l'obtention d'un contrat dépendant des ressources dont elles disposent et de leur conformité aux exigences du client. Recrutant sur poste ou sur affaire dans le cadre d'un appel d'offres, ou encore sur profil pour se constituer un vivier et anticiper un besoin potentiel, les SSII sont en permanence en quête de profils-types, auxquels les jeunes diplômés formés aux derniers langages sont les plus à même de répondre (Berrebi-Hoffmann, 2006). Mises en concurrence par les entreprises clientes, elles recherchent et se disputent les mêmes profils, créant ainsi un effet de rareté des compétences.

Dépendantes des clients et des jeunes diplômés, des SSII développent des pratiques déviantes (Curriculum Vitae truqués pour obtenir un marché, proposition au client de candidats qui ne sont pas encore embauchés...), qui expliquent leur mauvaise réputation. Qualifiées de " boîtes à régie ", de " marchands de viande », les SSII servent d'intermédiaire entre l'entreprise utilisatrice et le jeune diplômé et sont assimilées à une société d'intérim spécialisée dans les hauts niveaux de qualification ${ }^{7}$. Ces pratiques, préjudiciables aux salariés, sont rarement pénalisées afin de ne pas entraver le fonctionnement des SSII, propice à l'activité économique et au développement de l'emploi ${ }^{8}$. Entre droit et non droit, quid de l'impact de la relation de service sur la relation d'emploi ?

\subsection{L'ingérence du client et la triangulation des relations de travail}

La relation d'emploi unissant employeur et employé est traditionnellement bilatérale. Elle est encadrée par le droit du travail qui place le salarié sous l'autorité directe de l'employeur et lui accorde en contrepartie des garanties sociales. Ce lien de subordination reste la norme en vigueur ; cependant, le lien employeur/employé tend à s'affaiblir dans les configurations de travail où s'ingère le client, notamment dans le cadre d'externalisation d'activités. "La recomposition des systèmes productifs en chaînes et réseaux maillés de soustraitance " favorise en effet la triangulation des relations de travail entre client, employeur et salarié (Havard, Rorive, Sobczak, 2006). S'attachant à en établir la cartographie, les auteurs identifient plusieurs configurations selon la nature des relations économiques liant client et prestataire et selon la place que le salarié peut y occuper. Une entreprise cliente qui accorde sa confiance à son prestataire le laissera gérer en toute autonomie son équipe.

7. In « Délits de marchandage et prêts illicites de main-d'œuvre : le record en 2006 », 12/01/2007, Le Munci, association professionnelle des informaticiens.

8. Ibid. 
Mais quand les salariés d'un sous-traitant passent de fait sous la subordination du client qui les choisit, prescrit et contrôle leur travail, il y a dissociation entre " pouvoir de droit et pouvoir de fait " (ibid., 2006), entre "périmètre juridique et périmètre organisationnel " (Pichault, Rorive, 2003) et intrusion du client (donneur d'ordre, entreprise cliente). Le pouvoir d'ingérence du client est toutefois plus ou moins intrusif. Il l'est fortement dans des situations de mise à disposition de personnel pour des projets de longue durée, de prestations sur site de façon permanente (ibid.). Les pratiques d'externalisation de la main-d'œuvre au nom de "l'orientation client » affectent donc la nature et la régulation des relations d'emploi et de travail (Rorive, Gustavsson, 2006) renouant parfois « avec des formes anciennes (préfordistes ?) d'organisation du travail ", regroupées sous le terme de marchandage9 (Supiot, 1999, p. 47).

\section{Encadré 2 \\ Prêt ou trafic de main-d'œuvre?}

Le Code du travail français qualifie de marchandage toute opération à but lucratif ayant pour objet exclusif la fourniture de main-d'œuvre et parle de délit de marchandage quand elle cause un préjudice au salarié ou élude les dispositions légales ou conventionnelles (art. L. 8231-1). Le prêt de main-d'œuvre (ou mise à disposition de personnel) n'est pas illicite en soi. Il y a délit quand la prestation de service ou le contrat de sous-traitance se limite à placer de la maind'œuvre sous l'autorité d'un donneur d'ordre. La jurisprudence cherche dans la réalité des faits, non dans la formulation du contrat, s'il y a fourniture illicite de main-d'œuvre. Le prêt de maind'œuvre à but lucratif est réglementé et exclusivement réservé aux entreprises de travail temporaire. Dans la plupart des pays européens, la loi « distingue aujourd'hui le marchandage prohibé, ou trafic de main-d'oeuvre, de la sous-traitance ou de la prestation de services qui sont des contrats licites» (Supiot, 1999, p. 47).

La frontière entre le licite et l'illicite est en principe tracée et dépend de la nature et de la valeur ajoutée de la prestation fournie. Mais la multiplicité des formes contractuelles (sous-traitance, régie, mission) "n'aide pas à identifier facilement les modalités de déroulement des travaux demandés " (Rapport Chaudron, 2009). Dénonçant les usages abusifs des dispositifs de triangulation des relations de travail (intérim ou régie), l'auteur juge « inacceptable que des entreprises n'aient pour valeur ajoutée que le vivier de CV qu'elles ont à mettre à disposition de leurs clients " (p. 13). Cela n’a pas empêché le Cigref (club informatique des grandes entreprises françaises) ${ }^{10}$ de demander (sans succès), en 2010, l'abrogation de la loi sur le délit de marchandage! Pour clarifier la réglementation sur les

9. Par référence au " marchandeur » « qui convenait avec le client de l'ouvrage à réaliser " et le faisait réaliser par les ouvriers qu'il payait directement.

10. Créé en 1970 par les directions informatiques des grandes entreprises françaises. 
prestations intellectuelles ${ }^{11}$, le Syntec Informatique ${ }^{12}$ propose de substituer à la notion de mise à disposition celle de détachement dans le cadre d'un contrat commercial. Toutefois, ce n'est pas dans la nature du contrat mais dans le réel des conditions de travail que se joue la licéité du prêt de main-d'œuvre ; cela nous amène à distinguer les deux modes de prestation des SSII.

\subsection{Des pratiques d'emploi différentes selon les prestations de service}

Régie et forfait traduisent des conceptions différentes de la relation, de la prestation et de l'entreprise de service, déterminantes dans la définition de la relation d'emploi (Everaere et al., 2003).

Mode d'extériorisation de la main-d'œuvre, la régie (ou assistance technique) domine le marché des services informatiques et reste appréciée par les différents acteurs. Ce mode de facturation, basé sur un tarif journalier dont le montant varie selon le poste, présente de nombreux avantages. Pour le prestataire de service, l'activité est rémunératrice et la marge encaissée régulière et sans risque puisqu'elle dépend du nombre de jours travaillés. Pour le client, la régie répond à un besoin de souplesse et de compétences pointues. Pour les jeunes informaticiens, c'est le moyen d'accéder à des missions variées auprès de grands comptes, d'apprendre rapidement le métier, voire de se faire embaucher par le client. Au-delà de cette vision consensuelle, qu'on peut retrouver dans l'intérim (Belkacem et al., 2007), la régie est source de précarisation du travail (Stéphanon, 2007) quand l'enchaînement des missions, loin d'être progressif et de favoriser le développement des compétences, obéit à une logique marchande et d'évitement du coût des périodes d'inter-contrat ; en effet, dans ces conditions, le travail au sein de l'entreprise cliente ne favorise ni l'autonomie, ni l'initiative, ni l'intégration. Travailler au sein de collectifs auxquels il n'appartient pas conduit l'informaticien à surinvestir pour se faire reconnaître par les managers, faire face à la méfiance des salariés ou concurrencer les salariés d'autres SSII (Everaere, LapoireChasset, 2011). La formation étant quasi inexistante dans les petites SSII, le salarié doit assurer seul le maintien de son employabilité. Si un détachement de longue durée peut favoriser l'intégration chez le client, il rend difficile le passage à un travail en mode projet ou le retour au sein de la SSII (Everaere et al., 2003). L'informaticien peut aussi être confronté à des conditions de travail difficiles à cause du responsable commercial de la SSII ; ce dernier, chargé des relations avec le client, est parfois soucieux de conclure un accord, quelles qu'en soient les conditions pour l'informaticien. De telles pratiques distendent encore le lien avec la SSII. Plus globalement, le salarié en régie occupe une posi-

11. Source : Le journalduNet (28.03.2010).

12. Créé en 1970, Syntec informatique est la chambre professionnelle des sociétés de services et d'ingénierie informatiques (SSII) et des éditeurs de logiciels. Le Syntec informatique contribue au développement des technologies de l'information et de la communication, de leurs usages, assure la promotion des entreprises des logiciels et services, ainsi que la défense des intérêts collectifs professionnels. 
tion ambiguë, entre deux organisations, l'une qui l'emploie sur le plan juridique et lui confie une mission externalisée, l'autre, commanditaire d'un travail qu'elle est forcément amenée à contrôler ou évaluer. Il en résulte une ambiguïté juridique qui fait que le travailleur en régie, bien qu’en CDI (contrat à durée indéterminée) peut apparaitre moins protégé qu'un intérimaire. L'intérim est en effet le seul dispositif de triangulation des relations de travail encadré par le droit du travail. Celui-ci prévoit deux contrats : un contrat de mission entre l'intérimaire et l'entreprise de travail temporaire ; un contrat commercial, entre l'entreprise de travail temporaire et l'entreprise utilisatrice. Un intérimaire dont l'activité se poursuit au-delà du terme de sa mission voit son contrat de travail requalifié en relation d'emploi standard avec l'entreprise utilisatrice. Or, il n'est pas rare qu'un informaticien en régie travaille plusieurs années de suite chez un même client, au point de n'avoir guère de contact avec son employeur juridique, mais sans bénéficier des avantages des salariés de l'entreprise cliente, ni se voir proposer une embauche. Il se retrouve dans une situation "d'insider externalisé" (Everaere, Lapoire-Chasset, 2011).

Le forfait obéit à une tout autre logique. Parce qu'il vend un projet clés en main, de sa conception à sa réalisation, le forfait rétablit une relation partenariale entre prestataire et client, fondée sur la confiance et la négociation (des modalités, résultats et coûts de la prestation). Mais comme la SSII s'engage sur des résultats, des délais et des moyens dont l'anticipation peut être difficile s'agissant de nouvelles technologies, le travail au forfait constitue un risque : risque de dérive des coûts si les difficultés du projet ont été sousestimées par le commercial ou si le client demande une modification du projet initial sans révision de l'enveloppe de départ, risque de pénalités de retard en cas de performances insuffisantes de l'équipe. L'engagement demandé à l'équipe est certes plus fort (Everaere et al., 2003) mais parce qu'elle s'engage, la SSII garde la maitrise et le contrôle de ses ressources et maintient une relation d'emploi bilatérale avec ses salariés. Le travail au forfait requiert aussi une infrastructure plus importante (locaux, équipements) et des ressources stables ; bien mené, il est rémunérateur et permet de rentabiliser les investissements réalisés. Régie et forfait relèvent donc de choix stratégiques différents : recherche de profit à court-terme et chasse de jeunes diplômés vs souci de pérennité et construction d'une expertise spécifique. Toutefois, le recours à l'une ou l'autre de ces prestations semble lié à la conjoncture économique et aux fluctuations du marché. Le travail en régie se développe en période de crise (il est moins risqué que le forfait) ou quand la demande d'assistance est forte (passage à l'euro). Mais " asservi à la demande à court terme des entreprises et notamment des grandes " (Bret, 2005, p. 13), le fonctionnement en régie ne permet guère à une SSII de construire une stratégie propre sur le moyen terme, ni une image de marque.

A contrario, le marché du conseil et de l'infogérance ${ }^{13}$ revient en force (Bret, 2005), avec des externalisations de plus en plus intelligentes à traiter, supposant une offre globale et

13. Prise en charge de tout ou partie des ressources permettant de gérer une application informatique (personnel, locaux, matériel). 
intégrée pour des entreprises clientes aux exigences accrues. Enfin, entre régie et forfait, des variantes permettent aux SSII d'être moins dépendantes du client et de s'assurer des revenus réguliers en fournissant des prestations d'accompagnement et de maintenance, dans un cadre pluri-annuel. Le tableau 1 offre une synthèse des conditions de travail associées aux deux prestations.

Tableau 1

Conditions de travail et prestations

\begin{tabular}{|l|l|l|}
\hline \multicolumn{1}{|c|}{ Conditions de travail } & \multicolumn{1}{|c|}{ En régie } & \multicolumn{1}{c|}{ Au forfait } \\
\hline Régime d'emploi & $\begin{array}{l}\text { Mise à disposition de main-d'œuvre. } \\
\text { Logique de placement selon } \\
\text { opportunités }\end{array}$ & $\begin{array}{l}\text { Logique de projet négocié. } \\
\text { Logique d'intégration selon compétences }\end{array}$ \\
\hline Lieu de travail & Chez le client & SSIl - client \\
\hline Type d'intervention & $\begin{array}{l}\text { Individuelle, au sein d'une équipe de } \\
\text { salariés constituée par le client }\end{array}$ & $\begin{array}{l}\text { Collective, au sein d'une équipe constituée par } \\
\text { la SSIl }\end{array}$ \\
\hline Risque & $\begin{array}{l}\text { Difficultés d'intégration. } \\
\text { Surinvestissement pour se faire } \\
\text { reconnaître }\end{array}$ & $\begin{array}{l}\text { Pression forte, travail sous contrainte de délais et } \\
\text { de résultats }\end{array}$ \\
\hline $\begin{array}{l}\text { Organisation, } \\
\text { contrôle du travail }\end{array}$ & Client et sa hiérarchie & $\begin{array}{l}\text { SSII : chef de projet garant des engagements et } \\
\text { moyens, manager } n+1\end{array}$ \\
\hline Relation d'emploi & Triangulée & Bilatérale \\
\hline Lien salarié SSII & Faible & Fort \\
\hline
\end{tabular}

Source : Dietrich, Lauzier.

\section{2) Des SSII, lieux d'intégration professionnelle}

Dans nombre de SSII nouvellement créées, l'offre de services se résume à des prestations en régie, auprès de différents clients. Cette offre se diversifie généralement par la suite, d'autant que cette forme de prestations de service et d'emploi suscite des interrogations et des critiques de plus en plus nombreuses, au sein même de la profession, confrontée à des taux de turnover parfois très élevés. Le retour à des conventions au forfait semble à l'ordre du jour, aussi bien du côté des grandes entreprises informatiques (Alten, Cap Gémini) que des SSII de taille plus modeste, comme le montre l'exemple des deux SSII étudiées. Nous les présentons et analysons, ensuite, leurs pratiques de GRH et de management.

\subsection{Présentation des deux SSII}

Ces deux SSII different par leur taille, leur métier, leur positionnement stratégique et leurs choix de développement. L'une, I, est une société multi-sites de grande taille (1 000 salariés), spécialiste du "Maintien en conditions opérationnelles du système informatique ". 
L'autre, N, est une PME (petite et moyenne entreprise) indépendante de 220 salariés répartis sur quatre sites implantés près des clients. Elle est spécialisée dans la conception et le développement de solutions informatiques. Depuis leur création dans les années 90, les deux SSII connaissent une croissance forte et ininterrompue de leur chiffre d'affaires alors que le secteur des services informatiques a enregistré des baisses importantes à plusieurs reprises. Le chiffre d'affaires de I est passé de 2,9 à 57,9 millions d'euros en dix ans. L'entreprise est cotée en bourse. Le chiffre d'affaires de $\mathrm{N}$ est passé de 5 à 19,5 millions d'euros en dix ans. Les deux SSII ont eu pour stratégie de conforter leur position initiale et de renforcer leur expertise mais leurs choix de développement differrent.

\section{Encadré 3 \\ Les choix de I}

I s'est développé à partir de son métier de base pour devenir un spécialiste de la gestion technique des infrastructures informatiques (conception de l'architecture du système, apport de solutions et assistance dans l'utilisation au quotidien des outils informatiques). Ce choix l'a conduit à entretenir, dès le départ, des relations avec les grands comptes (EDF, BNP - Gaz de France, Banque nationale de Paris, ...) dotés d'une infrastructure informatique de pointe et demandeurs de ressources toujours plus performantes.

Pour y répondre, la SSII s'est dotée de structures de veille technologique et entretient des partenariats avec les grands éditeurs de logiciels. Pour prouver sa capacité à faire du volume et à proposer une offre complète de services garantissant aux utilisateurs un haut niveau de disponibilité, de sécurité et d'accessibilité à l'information, I a fait le choix d'une croissance externe, par rachats successifs de sociétés informatiques dont le métier est proche ou complémentaire de son métier de base (sécurité, infogérance).

Les grands comptes imposant le fonctionnement en régie, celui-ci représente $65 \%$ du chiffre d'affaires de I ; le reste (35\%) relève de prestations au forfait. La régie ne renvoie pas à une problématique purement financière pour I mais correspond à I'histoire de son développement. L'effort consenti pour équilibrer forfait et régie est loin d'être couronné de succès, notamment lorsque la stratégie de l'entreprise est d'assurer une croissance externe. Garder des clients comme les banques fournit à l'entreprise l'assise financière nécessaire pour assurer le maintien d'une activité propre à réduire les périodes d'inter-contrats.

En revanche, l'expérience et la renommée acquises auprès de ces grands comptes lui permettent aujourd'hui de se tourner vers de nouveaux marchés (industrie, administration publique, PME, associations) qui se révèlent être moins concurrentiels, comme le souligne le dernier plan stratégique (2008-2011). Le conseil permet à I d'accéder aux directions générales et par là même, à des missions plus importantes que celles proposées par les directions informatiques. La tendance forte des clients à externaliser la maintenance et les évolutions intervenues récemment sur le marché informatique font que le mode de facturation ne semble plus devoir être fixé au départ : il relève soit d'un projet, soit d'un contrat pluriannuel d'infogérance (DRH I). Le choix récent de créer des centres de services mutualisés (ensemble de services fournis par la SSII à différents clients dans le cadre de contrats forfaitaires de plusieurs années) doit contribuer à réduire le travail en régie, en permettant d'intervenir auprès d'une multiplicité de clients, sur la base du maintien de relations bilatérales d'emploi. 


\section{Encadré 4 \\ Les choix de $\mathrm{N}$}

Dès sa création, $\mathrm{N}$ a choisi de travailler au forfait, choix adapté à ses interventions en mode projet. Il représente 60 à $70 \%$ de son chiffre d'affaires, pour 30 à $40 \%$ en régie. Bien que le dirigeant en souligne les exigences et les risques pour la SSIl, le choix du forfait infirme l'idée selon laquelle les petites SSII ne peuvent travailler qu'en régie. Spécialisée dans l'ingénierie informatique, $\mathrm{N}$ développe, depuis 2002, des activités de conseil en maîtrise d'ouvrage en plein essor et, depuis $2006\left(^{*}\right)$, des activités de recherche (en lien avec des instituts comme l'Inria - Institut national de recherche en informatique et automatique -, Télécom) et d'innovation technologique (en lien avec le pôle de compétitivité des industries du commerce). Elles lui ont permis de faire face à la crise de 2008 et d'afficher une compétence distinctive en « informatique diffuse » ou mobile (associant téléphone mobile et systèmes d'information), avec la production de prototypes applicatifs pour la grande distribution et la vente par correspondance. L'entreprise est engagée dans la réalisation de d'importants projets utilisant les technologies émergentes et leur pilotage à partir de spécifications répondant au métier du client.

Innover est au cœur de sa stratégie pour fidéliser ses clients et assurer sa performance économique. Pour le dirigeant, accompagner le client c'est, au-delà de la compréhension de son besoin, anticiper et faciliter les évolutions de son métier : « la relation client-fournisseur doit s'inscrire dans la recherche d'une vision du métier. » $\mathrm{N}$ a donc fait le choix d'une croissance " interne et mesurée », conforme à son projet social de grandir tout en restant « une entreprise à taille humaine ».

L'innovation sociale constitue la marque de l'entreprise. Elle traduit le projet personnel du dirigeant (" être une référence en matière de progrès social (**) ») et fait de N une SSII atypique et médiatisée. Très engagé dans le développement d'un management social et environnemental responsable, ancien président du centre des jeunes dirigeants, figure médiatique dans sa région, le dirigeant est soucieux de garder son indépendance pour investir dans sa Fondation (engagée dans l'aide aux demandeurs d'emploi ou aux jeunes diplômés discriminés et aux pays en développement). II développe depuis peu des prestations de conseil et des solutions informatiques en management des ressources humaines et se tourne vers des clients qui partagent ces valeurs : en 2009, $60 \%$ de son chiffre d'affaires est réalisé dans le secteur de l'économie sociale et de la santé (CNAM - Caisse nationale d'assurance maladie -, Mutuelles...).

$\left(^{*}\right)$ : Cette volonté de « devenir leader des nouvelles technologies dans la région » n'est pas nouvelle et s'inscrit dans la durée (Informatique, $\mathrm{n}^{\circ}$ 1545, 28 mai 1999).

$\left(^{* *}\right)$ : Rapport d'activité 2009.

Les dirigeants de ces deux SSII affichent une volonté constante d'assurer la visibilité de leurs savoir-faire sur un marché très concurrentiel ; cela se traduit par la conquête et la collection de trophées, techniques pour l'une, managériaux pour l'autre ${ }^{14}$. Pour ces sociétés, s'assurer d'une crédibilité auprès de clients potentiels passe par des performances

14. De nombreux trophées, remportés au cours des années 2000, attestent de capacités d'innovation en matière technique et managériale pour $\mathrm{I}$, de préoccupations sociales et managériales pour $\mathrm{N}$. 
économiques et financières remarquées et saluées dans le secteur d'activités, et parfois audelà. L'objectif est double : d'une part, assurer la pérennité de la relation avec les clients en renforçant sa position sur le marché ; d'autre part, se faire connaitre auprès d'autres clients. "Faire comprendre par des actes qui on est ", cela vaut autant pour les clients que pour les salariés (Dirigeant de $\mathrm{N}$ ).

\subsection{Le choix d'investir dans les ressources humaines}

Les deux SSII affichent leur volonté de construire une véritable politique de gestion des ressources humaines. Leurs stratégies de recrutement visent à ne pas faire de la compétence informatique le seul critère de sélection et à diversifier les profils de leurs collaborateurs.

On distingue deux types de recrutements : conjoncturels et structurels. Les premiers s'inscrivent dans le court terme et sont soumis aux contraintes du marché et aux exigences du client. Les seconds visent à maintenir un haut niveau d'expertise et à renforcer l'encadrement. Ils ne sont possibles que si l'entreprise a une certaine visibilité sur son activité future, ce qui suppose un marché en développement, avec des projets exigeant la constitution d'équipes et de longs mois de travail collectif.

Les deux sociétés ont donc choisi de diversifier leurs sources de recrutement : l'une (I) a investi sur un haut niveau de diplôme (bac +8 ), avec l'embauche de docteurs sans perspective sur le marché de l'emploi et sur des profils expérimentés, avec 5 à 10 ans d'expérience. Lautre (N) s'est engagée dans un management de la diversité (pratique du CV anonyme) et recrute des profils centrés sur le métier du client (masters santé) pour des postes de consultants en maîtrise d'ouvrage ou des informaticiens de moindre niveau (licence professionnelle) qu'elle forme en interne et fidélise plus facilement. Les deux SSII tirent aussi profit des licenciements collectifs de grandes entreprises pour recruter des cadres informatiques de 40-45 ans comme chefs de projet. Ces stratégies leur permettent d'une part, de se doter d'expertises différenciées, propices à la diversification des activités; d'autre part, d'établir une relation durable avec des candidats dont le parcours a souvent été marqué par une forte mobilité, assumée ou recherchée par certains, avant d'être subie, voire assortie de périodes de chômage pour les plus âgés.

Un des collaborateurs (DUT - diplôme universitaire de technologie - « informatique ») interrogés chez I retrace un parcours fait d'une succession de CDD (contrat à durée déterminée) au sein de collectivités locales comme adjoint au directeur des systèmes informatiques, puis directeur de service informatique dans les services de villes franciliennes d'une importance croissante, avant de rejoindre I :

"J'avais des contrats de 3 ans, jétais marié, je me suis dit, il faut que j'attrape un CDI. J'ai trouvé I, c'était la première société à me proposer un entretien, le directeur commercial m'a tendu un contrat de 15 jours. Je suis allé aux autres entretiens, ceétait des tests délirants et j'avais 10 ans d'expérience. J'avais bien aimé le côté relationnel chez I". 
Loin d'avoir eu un parcours aussi linéaire, une autre salariée (bac +2 en histoire) fait état d'une succession de missions, dont la durée pouvait varier de quelques mois à 2 ou 3 ans, successivement comme ingénieur d'affaires au sein d'une PME, puis dans un groupe international de communication/évènementiel, avant de devenir chef de projet à $\mathrm{Fr} 3$ puis au crédit coopératif, pour finir embauchée par I. Le profil de ces nouveaux embauchés est recherché par la SSII à la fois pour les compétences qu'ils peuvent mettre en œuvre chez le client et pour leur expérience non seulement technique mais relationnelle ; à charge pour l'encadrement de prendre en compte leur desiderata :

"La première mission ne me convenait pas, 6 mois à administrer un ERP (progiciel de gestion intégré) chez Alsthom. C'est moi qui ai demandé à changer, c'était le stress absolu. La mission était très difficile, une usine avec une CGT très dure et les informaticiens me mettaient des bâtons dans les roues. La seconde mission, chez Alcatel, (j'y suis encore)" (Technicien).

C'est en leur offrant la possibilité de se former et d'évoluer professionnellement que les deux SSII s'efforcent de retenir leurs collaborateurs. Pour les fidéliser, elles mobilisent des moyens comparables : parcours d'intégration, principes d'une organisation apprenante fondée sur le partage d'expériences, analyse des bonnes pratiques internes et externes, réalisation d'études prospectives mobilisant veille et recherche. Elles ont toutes deux créé une université d'entreprise pour renforcer les compétences techniques et méthodologiques requises par les métiers de l'entreprise et assurer la transmission de la culture et des valeurs de l'entreprise. $\mathrm{N}$ a aussi créé une école des compétences (2008) relatives à la gestion de la relation client (encadrement, médiation, négociation). Des parcours de formation par filières réunissant les collaborateurs d'un même métier (ingénieurs, consultants, chefs de projet) mais de tous niveaux (juniors, seniors) favorisent le partage d'expériences et le développement de collectifs autour de "la façon de faire son métier " (DRH N). $43 \%$ des collaborateurs ont déjà suivi cette " école de facilitation " et témoignent, lors d'enquêtes, de leur satisfaction. Ils soulignent la "prise de conscience de comportements implicites : on se rend mieux compte de ce qu'on fait naturellement de bien ou de moins bien... On s'aperçoit quion a les mêmes problèmes et qu'on peut trouver des solutions ensemble " (chef de projet informatique). Ces parcours de formation s'articulent à des parcours professionnels susceptibles de répondre aux ambitions de carrière des salariés chez $\mathrm{N}$. Un outil de gestion informatisé " décrit clairement toutes les étapes d'un parcours professionnel sur plusieurs années, les moyens de progression et de formation, les points d'avancement $R H$ " (rapport activités 2009). Cet outil est désormais commercialisé et $\mathrm{N}$ offre des prestations en $\mathrm{RH}$, dans le cadre d'un Institut du management et des RH chargé d'accompagner les clients.

Aux collaborateurs qu'elle veut garder, I propose un parcours dont la première étape passe par l'accès au statut cadre. La fonction cadre correspond à un certain niveau de responsabilités, d'abord au sein de la SSII : "En SSII, il y a des métiers sans prise de décision, comme pilote d'installations, c'est une fonction d'exécution, ou encore technicien de micro, et des postes nécessitant de la réflexion comme chef d'équipe ou chef de projet qui consiste à fournir de la documentation et faire le suivi du projet et des collaborateurs... encore faut-il demander à 
passer cadre. Demander à passer cadre, c’est penser à son avenir ", comme le déclare un collaborateur. Mais le passage au statut cadre peut s'opérer également à l'issue d'une forte évolution chez le client, car "si le collaborateur évolue chez le client, c'est un bon collaborateur". C'est le parcours suivi par un de ses collègues : «Pour le client, je suis expert dans le domaine Oracle... je suis le garant de solution technique pour le client... Et je suis resté à I comme consultant... ça s'est fait en 2 étapes, ce passage à cadre : j’avais des augmentations régulières en rapport avec ce que je faisais chez mon client avec des collègues comme DBA international (Database Administrator), j'ai été voir mon commercial, je lui ai dit: "jai fait mes preuves, je veux une augmentation conséquente". I a décidé que jétais un profil quelle soubaitait garder, j'ai eu mon augmentation. "Deux voies d'évolution professionnelle s'offrent donc au collaborateur passé cadre. Il occupe d'abord une fonction non hiérarchique de responsable technique délégué au sein de son équipe. Soit il choisit de développer une expertise technique, à l'issue de changements successifs de fonction chez le même client, à l'exemple du collaborateur passé DBA expérimenté puis senior. Soit il accepte des fonctions de manager en interne : c'est le cas pour un collaborateur nommé successivement chef de projet (2005), responsable opérationnel de compte client (2008), et responsable d'un centre de services mutualisés (2010). Ces possibilités d'évolution interne sont d'autant plus nombreuses que la SSII a acquis une taille et une notoriété suffisantes pour détenir un portefeuille clients important.

\subsection{Créer des relations de proximité : un souci commun}

Les deux SSII ont développé un réseau de proximité pour compenser l'éloignement géographique et l'isolement des collaborateurs chez le client. Quel que soit leur parcours, les salariés de I rencontrés, embauchés à bac +2 , déclarent ne pas vouloir quitter la SSII, même si le taux de turnover reste pour l'entreprise dans la moyenne : "Mon patron (le client) essaie de m’intégrer, mais je suis bien à I, pour le même salaire, je n'aurais pas ma qualité de relations avec mon PDG, le DRH. Perdre ce relationnel pour une boîte qui fait charrette tous les ans? Non. Quand on est mal, on accepte. Mais je suis bien chez I. ici, ce n'est pas la guerre, c'est sain, on connaît le patron, on fait attention quand je dis 'jai Gh de route'. ». Un autre de renchérir : "L'avenir ? À court terme, c'est I., dans 2 ou 3 ans, aussi. À 10 ans, je ne sais pas. C'est une bonne boîte et j'ai été bien reçu... Non, je n'ai pas le projet de changer d'entreprise. On est plus suivi. On peut écrire à la DRH, elle répond. L'aide technique, c'est plus le client qui la fournit... I c'est plus le salaire et la formation, c'est pas pour les problèmes techniques ou le suivi du travail. Le référent, c'est le responsable d'agence".

Ces propos sont d'autant plus significatifs qu'il s'agit de travailleurs en régie. Chez I, un manager assure le suivi des collaborateurs au sein de chaque équipe projet. Mais sa taille croissante a contraint la SSII à proposer à des managers de terrain une mission de responsable technique délégué (RTD), pour l'encadrement de proximité. En charge de la gestion d'équipes projets, le RTD doit acquérir une connaissance précise des besoins du client mais aussi gérer le suivi des collaborateurs ; à cet égard, il doit s'assurer de l'accueil qui 
leur est réservé chez le client, et en tant que représentant de la SSII, il doit entretenir avec chacun d'eux une relation de proximité, sans avoir pour autant de fonction hiérarchique.

$\mathrm{N}$ enregistre un taux de turnover faible par rapport au secteur. $\mathrm{N}$ a renforcé l'équipe $\mathrm{RH}$ et le management de proximité. L'activité en mode projet respecte l'autonomie des collaborateurs. Elle favorise en outre le travail en équipe autour d'une hiérarchie de projets plus latérale que verticale car fondée sur l'expertise et la conception d'offres à forte valeur ajoutée. Au-delà du chargé d'affaires et du chef de projet encadrant l'activité de l'équipe, un manager sans lien opérationnel gère le suivi professionnel de huit salariés et les informe des projets à venir afin qu'ils puissent les intégrer. La fonction RH intervient en appui, avec un rôle de conseil et de coach tout au long du parcours professionnel. Soucieuse d' " être la SSII où l'on est le mieux pour travailler ", $\mathrm{N}$ a confié à la DRH la définition et la construction d'un référentiel de "l'entreprise à taille humaine». La proximité avec la direction et la DRH est plébiscitée, de même que la réactivité de l'entreprise dans le suivi régulier des salariés et la volonté de prendre en compte les contraintes personnelles (déplacements, charges familiales) et de faciliter le règlement des contraintes administratives (frais divers, véhicule de fonction, etc.).

Enfin, au-delà des attentes en matière d'accompagnement, les deux SSII favorisent la communication interne et externe ; l'organisation de rassemblements contribue au développement d'un sentiment d'appartenance et d'un mode de management fondé sur « la densité du tissu et des échanges sociaux " (Berrebi-Hoffmann, 2006, p. 166). Les deux SSII font ainsi état de la personnalisation des liens qu'elles s'efforcent de créer avec leurs salariés, non seulement à travers des entretiens réguliers d'évaluation mais en organisant une série d'évènements fédérateurs auxquels les salariés sont conviés à participer activement : soirées recrutement pour I, séminaire collectif annuel et groupes de travail sur la stratégie d'entreprise pour $\mathrm{N}$, week-end festifs, implication des salariés dans la fondation de N, enquêtes internes sur la satisfaction des salariés ou leurs attentes, création d'un cadre de vie favorisant l'équilibre entre vie professionnelle et vie personnelle. En 2000, N s'est installé à la campagne pour favoriser le bien-être au travail et I, située en Ile-de-France, envisage de favoriser la mobilité vers ses filiales en province pour ceux qui le souhaitent. C'est une autre façon de densifier le réseau d'échanges entre pairs auquel ces travailleurs autonomes se disent particulièrement sensibles.

\section{Conclusion}

Sur un marché hautement concurrentiel, les pratiques des SSII, et plus généralement du secteur des services informatiques, témoignent de l'impact que peut avoir le client sur la gestion d'une entreprise prestataire : celui-ci peut aussi bien peser sur la définition de son métier que sur les conditions de travail de ses salariés. Mais malgré les contraintes imposées par les clients, notamment par les grands comptes, les entreprises étudiées montrent 
que des choix sont possibles pour élaborer une stratégie à moyen et long terme. Sa mise en œuvre nécessite l'établissement de relations durables avec un certain nombre de collaborateurs, notamment de l'encadrement, et une véritable politique de gestion des ressources humaines : celle-ci consiste non seulement à recruter, mais à évaluer, à former et à proposer des perspectives de carrière. Dans ce type d'entreprises, la régie ne constitue pas nécessairement une forme précaire d'emploi, comme en témoignent les entretiens menés avec les salariés : il n'existe donc pas de lien automatique entre types de prestation de service (régie/forfait) et modalités d'emploi (précaire/stable).

À la différence des grandes entreprises du secteur, les deux SSII ne recrutent pas prioritairement des jeunes ingénieurs en informatique. Elles favorisent plutôt une diversité de profils (niveaux de diplômes, domaines de compétences, champs d'expérience) dont le maillage est fécond pour l'entreprise. Les nouveaux embauchés bénéficient d'une stabilité d'emploi. Les informaticiens expérimentés, touchés par le chômage, y trouvent de nouvelles opportunités de développement dans la mesure où les dirigeants ont besoin d'un encadrement stable et de compétences collectives stabilisées. Les choix faits par ces SSII de mieux équilibrer forfait et régie permettent aux salariés de choisir les missions et les modalités de travail qui leur conviennent, entre régie et forfait, expertise ou management. Ces deux SSII peuvent certes apparaître atypiques, face aux discours dominants dénonçant la précarisation des hauts niveaux de qualification sur le marché des services informatiques. Pour autant, elles traduisent des préoccupations communes aux grandes SSII, soucieuses de renouveler leur image en développant des pratiques de GRH susceptibles de retenir leurs salariés et de renforcer un lien de proximité conforme aux attentes des professionnels de l'informatique.

\section{Bibliographie}

APEC (2006), "Les pratiques de recrutement des SSII ", Bilan statistique et enquête qualitative, Département Etudes et Recherche, août.

Belkacem R., Kornig C., Michon F. (2007), " Les origines du travail intérimaire et son développement en Europe ", Xlème Journées Internationales de Sociologie du travail, Londres.

Boltanski L., Chiapello E. (1999), Le nouvel esprit du capitalisme, Paris, Gallimard.

Baret C., Everaere C. (2002), « Le modèle individualisant de GRH à l'épreuve dans les sociétés de conseil en informatique, une comparaison France, Allemagne, Pays Bas ", XIIIème Congrès de l'AGRH, Nantes, pp. 83-92.

Berrebi-Hofmann I. (2006), "Les consultants et informaticiens. Un modèle d'encadrement de professionnels à l'échelle industrielle ", Revue Française de Gestion, n 168-169, pp. 157-176. 
Bret C. (2005), " L'histoire des 40 premières années des SSII à travers leurs hommes et leurs activités ", Entreprises et Histoire, n 40, pp. 9-19.

Chaudron T. (2009), Les tiers employeurs, ou comment conjuguer compétitivité et responsabilité dans la France du XXI siècle, Rapport au ministre du Travail, des Relations sociales, de la Famille, de la Solidarité et de la Ville, février.

Everaere C., Grimand A., Laviolette M. (2003), "Formation, conditions de travail et relations sociales dans les sociétés de services informatiques ", Education Permanente, $\mathrm{n}^{\circ} 155$, pp. 147-155.

Everaere C., Lapoire-Chasset M. (2011), "le salarié mis à disposition : un insider externalisé ", Actes du XXIIème Congrès de l'AGRH, Marrakech.

Fondeur Y., Sauviat C. (2003), « Les services informatiques aux entreprises : un marché de compétences ", Formation Emploi, nº 82, pp. 107-132.

Gazier B. (2006), "L'employabilité ", Encyclopédie des Ressources Humaines, Allouche J. (coord.), $2^{\text {ème }}$ Edition, Paris, Vuibert.

Havard C., Rorive B., Sobczak (2006), «Client, Employeur et Salarié : cartographie d'une triangulation complexe, Economies et Sociétés, AB, n 27, pp. 1229-1258.

Lanciano-Morandat C. (1996), "Les ingénieurs des sociétés de services et d'ingénierie informatique ", Formation Emploi, n 55, pp. 77-89.

Liaroutzos O., Robichon M. (1999), " La recomposition des métiers de base de l'informatique ", Bref Cereq, n ${ }^{\circ} 155$.

Pichault F., Rorive B. (2003), «Restructurations et nouveaux périmètres organisationnels : le rôle de la fonction RH », Journées d'études, GREGOR-IAE de Paris, 27 fév.

Poussou-Plesse M., Perrin Joly C., Duplan D. (2010), «La sélection par l'âge dans les métiers informatiques, l'adaptabilité en question ", Travail et Emploi, n 121, pp. 21-32.

Poussou-Plesse M., Duplan D., Guillemard A.M., Perrin Joly C. (2009), Durer au travail, dans les métiers de l'informatique, Rapport pour la Dares.

Rorive B., Gustavsson E. (2006), Les relations de travail en Europe. D'une conception bipolaire à une conception triangulaire voire multipolaire. Diversité et conséquences de l'orientation client sur l'entreprise et ses acteurs, Rapport transversal.

Simula P., Roualdes D. (2002), "Les prestataires de services informatiques », BrefCereq, $n^{\circ} 189$.

StÉPHANON P. (2007), « Précarité de l'emploi et précarité du travail : le cas des intérimaires », XIème Journées Internationales de Sociologie du travail, Londres.

Supiot A. (dir.) (1999), Au-delà de l'emploi, transformations du travail et devenir du droit du travail en Europe, Rapport pour la Commission européenne, Paris, Flammarion. 
SynteC (2006), Pour une vision prospective à cinq ans des métiers de la branche, Syntec-CICF. Zune M. (2003), "Technologies de l'information et de la communication : quelles logiques professionnelles? ", Formation Emploi, n 82, pp. 11-21.

Zune M. (2006), « De la pénurie à la mobilité : le marché du travail des informaticiens », Formation Emploi, pp. 5-24. 